\title{
Importancia de la marca paraguas y el made in en la competitividad internacional del granito
}

\section{Brand umbrella and made in the international competitiveness of granite}

\author{
Ma José García Rodríguez ${ }^{1}$ \\ José Santiago Gómez Fraiz ${ }^{1}$ \\ Ana Isabel Martínez Senra ${ }^{1}$ \\ Universidad de Vigo
}

Recibido el 26 de marzo de 2009, aceptado el 30 de abril de 2010

$\mathrm{N}^{\mathrm{o}}$ de clasificación JEL: M31

DOI: $10.5295 / \mathrm{cdg} .100153 \mathrm{mg}$

Reseña Bibliográfica: GARCÍA RODRÍGUEZ, M. J.; GÓMEZ FRAIZ, J. S.; MARTÍNEZ SENRA, A. I. (2010): "Importancia de la marca paraguas y el made in en la competividad internacional del granito"; Cuadernos de Gestión, Vol. 10. № 2, pp. 29-42. DOI: 10.5295/cdg.100153mg

\section{Resumen:}

España, al igual que Italia, líder tradicional del sector de granito, ha visto como China, India o Brasil, han escalado posiciones en el ranking de producción y exportación mundial de granito. En un contexto globalizado, es necesario posicionarse frente a estos competidores en los mercados internacionales, y dado que estamos ante un producto genérico que cumple unas condiciones adecuadas de precio y calidad, una forma de identificarlo y diferenciarlo es aportándole valor mediante la creación de una marca. En el trabajo se analiza la utilidad de una estrategia basada en el made in para el caso de Galicia, núcleo fundamental de la industria en España, alternativa que resulta interesante para las empresas consultadas pero cuya puesta en práctica exige la colaboración entre éstas y las instituciones.

Palabras clave:

Granito, marca, made in, internacionalización.

\begin{abstract}
:
Spain, like Italy, the traditional leader of granite sector, has seen how China, India or Brazil have risen several positions on global production and export rankings. In a globalized context, it's necessary a good differentiation from international competitors, much more when this is a generic product that offers suitable prices and quality. One way to identify and differentiate it, is providing value by creating a brand. In this paper we study the utility of a strategy based on made in for Galicia, major production location for the industry in Spain; an alternative that seems attractive to enterprises, but whose implementation requires strong collaboration between firms and public and private institutions.
\end{abstract}

Keywords:

Granite, brand, made in, internacionalization.

\footnotetext{
${ }^{1}$ Departamento de Organización de Empresas y Marketing- Universidad de Vigo. Facultad de Ciencias Económicas y Empresariales. Lagoas Marcosende s/n- 36310 Vigo (Pontevedra). E-mail: mjgarcia@uvigo.es. jsgomez@uvigo.es. aimtnez@uvigo.es
} 


\section{INTRODUCCIÓN: IMPORTANCIA DE LA MARCA Y EL MADE IN EN EL PROCESO DE INTERNACIONALIZACIÓN}

La globalización de la economía obliga a las empresas a competir en un entorno internacional cada vez más complejo, en el que la evolución de la tecnología y las comunicaciones se refleja en unos productos con componentes tangibles cada vez más estandarizados que dificultan su diferenciación. A su vez, los consumidores son más exigentes y la información disponible supone un factor importante en el proceso de decisión de compra.

En este sentido se ha comprobado que cuando existen creencias muy arraigadas sobre un producto, éstas constituyen los elementos básicos sobre los que un consumidor basa su conducta, por tanto, es importante conocerlas para diseñar estrategias de mercado (Rice, 1993 $)^{1}$. Así, la realización de una compra puede verse influida por el lugar de procedencia del producto o made in (Bilkey y Nes, 1982), entendido como toda influencia, prejuicio, predisposición, inclinación o predilección de los consumidores por el origen de un producto. Se trata de un atributo extrínseco e intangible (Hong y Wyer, 1989) además de multidimensional, puesto que influye en el consumidor en base a todas las dimensiones que éste pueda identificar en él, como la historia, cultura, desarrollo económico y político o la capacidad de comercialización (Ibáñez y Montoro, 1996).

El efecto del país de origen se ha analizado desde diferentes perspectivas y con varias metodologías. Como conclusión general se coincide en que el país de origen influye en el proceso de decisión de compra dependiendo de la cantidad de información del consumidor y del poder de diferenciación de los otros atributos. A partir de esta conclusión se desarrollan dos teorías (Han, 1989): 1) El efecto Halo que se basa en convertir la imagen del lugar de procedencia de un producto en un identificador del mismo, cuando por falta de información o por la dificultad para diferenciar otros atributos el conocimiento del producto es escaso (Johannson et al., 1985 y Erickson et al., 1984); 2) El efecto Summary basado en que cuando existe información y se pueden diferenciar otros atributos del producto, el made in se valora como uno más (Han, 1990) y su influencia estará condicionada según se trate de una categoría de producto conocida por el consumidor (Heimbach et al., 1989), sea una marca conocida (Han y Terpstra, 1988 y Cordell, 1992) o por la información que exista del producto (Han y Terpstra, 1988 y Hong y Wyer, 1989).

En productos procedentes del exterior, el made in adquiere un mayor interés debido a que los clientes se encuentran menos familiarizados con ellos. En este caso, puede suponer un elemento de referencia para los consumidores que se podrá aprovechar siempre que vaya acompañado de una imagen exterior positiva del país, contribuyendo a personalizar los productos y a identificarlos (Valls, 1992). Está demostrado que la existencia de una imagen positiva influye en una mejor valoración y en la intención de compra (Bilkey y Nes, 1982; Papadopoulos y Heslop, 2002).

\footnotetext{
${ }^{1}$ Las creencias son el antecedente directo de las actitudes en los modelos de formación de intenciones. Si actuamos sobre ellas podemos reconducirlas para conseguir conductas deseables, tanto en el proceso de decisión de compra como en la comunicación comercial (Mackenzie, Lutz y Belch, 1986). Sin embargo, cuando van asociadas a una mala imagen del producto, afectarán negativamente a la demanda.
} 
No obstante, una imagen consolidada como país puede contribuir a la exportación de sus productos, también es importante el papel que juegan las marcas notorias que destacan su origen, en tanto que contribuyen a la formación de una imagen positiva de éste en los mercados internacionales. La marca puede suponer una valiosa herramienta para conseguir diferenciarse de los competidores y realiza una doble función de identificación y diferenciación ${ }^{2}$.

La estrategia comercial consistente en la diferenciación del producto a través de la marca intenta conseguir que el cliente perciba características especiales en éste que lo distingan del resto de los productos sustitutivos y, además, que valore estas características, en su mayor parte intangibles. Si conseguimos diferenciar el producto obtendremos una base de clientes fieles; como resultado, las empresas con una marca reconocida o notoria consiguen un mayor nivel de competitividad.

El país de origen y la marca intercambian su protagonismo en el ciclo de vida del producto (Niss, 1996). Así, cuando un producto está empezando a conocerse en el mercado, el lugar de procedencia es la información que ofrece mayor seguridad para el consumidor y, por tanto, se convierte en un elemento de diferenciación durante las fases de introducción y crecimiento; posteriormente, la marca comienza a ser el punto de referencia para los clientes y se convierte en la protagonista (Lampert y Jaffe, 1998). A partir de este momento, las marcas pueden contribuir a la imagen del país de origen y, a su vez, ayudar a otras en su proceso de internacionalización (Montaña, 1993).

El activo país de origen es más complejo que la marca debido a que éstas se producen en países y aunque tengan un valor positivo en el mercado, puede que el país tenga un valor negativo. Asimismo, las marcas que se fabriquen en el extranjero pueden ver perjudicada su imagen por la del país donde se producen. Por este motivo, hay que analizar cuáles son las imágenes asociadas a un país y cómo pueden afectar a las percepciones de calidad y al proceso de decisión de compra.

De todos modos, no debemos olvidar que la marca país puede ayudar a vender el producto pero ha de ir acompañada siempre por un respaldo de calidad y un precio adecuado 3 (Kaynak y Cavusgil, 1983; Knight, 1999) porque, en caso contrario, también se podría perjudicar la imagen de los productos.

Se ha comprobado que los países con una buena imagen exterior suelen tener marcas reconocidas en los mercados internacionales que utilizan el país de origen dentro de su estrategia de comunicación, contribuyendo al refuerzo de esa imagen, tal y como ocurre en Alemania, Japón o Italia (Oporto del Olmo, 1993). Por tanto, con el paso del tiempo surgen unos beneficios recíprocos entre la imagen del país y sus marcas, contribuyendo a su credibilidad y seguridad, esto se conoce como «fertilización cruzada» (Álvarez del Blanco, 2000; Laroche et al., 2005).

\footnotetext{
${ }^{2}$ De todos modos, la importancia de la imagen de marca también dependerá del tipo de producto (Lampert y Jaffe, 1998).

${ }^{3}$ Los consumidores menos expertos pueden verse influidos por el made in, mientras que los más expertos y con mayores conocimientos sobre el producto basan sus evaluaciones en los atributos, sobre todo, en los aspectos más técnicos y específicos (Cerviño, 2002, p.157).
} 
Las instituciones deben desarrollar políticas de gestión de la imagen país que contribuyan a su reconocimiento en el exterior (Valls, 1993) ${ }^{4}$. No obstante, también es necesario un papel activo por parte de las empresas, que deben establecer mecanismos coordinados con las instituciones, además de incorporarlos en sus planes de marketing (Parameswaran y Pisharodi, 1994; Lin y Chen, 2006) ${ }^{5}$.

Una vez planteada la importancia de la marca y el made in para competir en el mercado internacional, y dado que en la actualidad las empresas graniteras españolas no están aprovechando sus beneficios, en el presente trabajo se hace un análisis del sector para valorar las posibilidades de creación de una marca paraguas reforzada por el made in.

\section{SITUACIÓN DEL SECTOR DE LA PIEDRA NATURAL}

En el sector de la piedra natural (granito, mármol y pizarra) el liderazgo que ocupó Italia hasta la década de los 90 ha dejado paso a las grandes explotaciones de China, India e Irán. Brasil y Turquía también han aumentado de forma espectacular su producción y están amenazando con desplazar aún más a Italia. Estos países se caracterizan por la disponibilidad de grandes recursos geológicos, una mano de obra con costes inferiores a los de la Unión Europea, una normativa medioambiental menos exigente y por la incorporación de tecnología punta durante los últimos años.

España también se ha visto muy afectada por el crecimiento experimentado por estos países, como podemos observar en los tablas 1 y 2 , donde se refleja la pérdida de posiciones de estos últimos años en producción y exportación de piedra natural, pasando de la segunda a la sexta posición en el ranking de países productores y del segundo al quinto puesto en volumen de exportaciones.

Tabla 1

Principales países productores de piedra natural (miles de toneladas)

\begin{tabular}{l|c|l|c}
\hline \multicolumn{1}{c|}{ Países } & Año 1990 & \multicolumn{1}{|c}{ Países } & Año 2005 \\
\hline Italia & 7.250 & China & 20.000 \\
\hline España & 3.250 & India & 12.500 \\
\hline Grecia & 1.850 & Irán & 10.400 \\
\hline
\end{tabular}

\footnotetext{
${ }^{4}$ Se ha demostrado que una buena imagen exterior facilitará la participación de las empresas en los mercados internacionales por los siguientes motivos: porque el origen de un producto no actúa como barrera de entrada, un alto nivel de reconocimiento en los mercados exteriores contribuye a una mayor credibilidad frente al consumidor y cuanto mayor sea la oferta podrán competir en mejores condiciones porque la presencia en los mercados exteriores será mayor (Baker y Ballington, 2002; Laroche et al., 2005).

${ }^{5}$ Conviene tener en cuenta que, aunque las empresas puedan beneficiarse a la hora de comercializar sus productos de una buena imagen de made in, puede ocurrir que hagan un mal uso de ésta ofreciendo productos de calidad inferior (Argawal y Sikri, 1996). Esto puede perjudicar tanto a la imagen del país de origen como a otras organizaciones que comercialicen sus productos apoyadas en ella, lo cual hace indispensable que el sector establezca un sistema de vigilancia para realizar un seguimiento continuo de cómo perciben la imagen los consumidores y que permita desarrollar las acciones necesarias para corregirlo.
} 


\begin{tabular}{l|c|l|c}
\hline \multicolumn{1}{c|}{ Países } & Año 1990 & \multicolumn{1}{c}{ Países } & Año 2005 \\
\hline EEUU & 1.700 & Italia & 10.140 \\
\hline Francia & 1.100 & Turquía & 8.250 \\
\hline China & 1.000 & España & 7.987 \\
\hline Brasil & 900 & Brasil & 6.900 \\
\hline Turquía & 550 & Portugal & 3.000 \\
\hline Alemania & 300 & Egipto & 2.500 \\
\hline Japón & 250 & Grecia & 2.100 \\
\hline
\end{tabular}

Fuente: Roc Natural Stone in the world 2007

Tabla 2

Principales países exportadores de piedra natural (miles de toneladas)

\begin{tabular}{l|c|l|c}
\hline \multicolumn{1}{c|}{ Países } & Año 1990 & \multicolumn{1}{c}{ Países } & Año 2005 \\
\hline Italia & 2.200 & China & 9.943 \\
\hline España & 730 & India & 3.566 \\
\hline India & 630 & Italia & 3.124 \\
\hline Sudáfrica & 610 & Turquía & 3.067 \\
\hline China & 500 & España & 2.422 \\
\hline Corea del Sur & 464 & Brasil & 2.119 \\
\hline Brasil & 440 & Croacia & 1.254 \\
\hline Portugal & 450 & Portugal & 1.210 \\
\hline Francia & 270 & Egipto & 972 \\
\hline Finlandia & 242 & Bélgica & 962 \\
\hline
\end{tabular}

Fuente: Roc Natural Stone in the world 2007

En el año 2005 China es líder en la producción y exportación de piedra, representando un $21,13 \%$ de la producción mundial y un 27,34\% de la exportación (en el año 1990 estos porcentajes eran sólo del 3,45\% y 5,91 \%, respectivamente), desbancando a Italia de la primera posición ${ }^{6}$. Además de tener las mayores reservas mundiales, sus productos no sólo están entrando a precios muy bajos y con una materia prima de calidad en nuestras fronteras, sino que están absorbiendo mercados que tradicionalmente habían pertenecido a Europa.

Otros países como India e Irán han aumentado de forma considerable su producción. Mientras que en el año 1990, no aparecían entre los diez principales productores, en el

\footnotetext{
${ }^{6}$ En el año 1990 Italia representaba un 24,78\% de la producción mundial de piedra natural. En el año 2005 este porcentaje se reduce al $10,71 \%$.
} 
2005 ocupan la segunda y tercera posición (tabla 1). Turquía ${ }^{7}$ también ha multiplicado por 15 su volumen de producción.

Los cambios registrados en el sector de la piedra natural se reflejan en el sector del granito, donde España es la tercera potencia en producción mundial tras China e Italia, procediendo el $80 \%$ de su granito ornamental de Galicia, que es la principal Comunidad exportadora, tanto en peso como en valor. Analizando la producción y exportación de granito (tabla 3) se observa un continuado descenso de las exportaciones desde el año 1996, explicado por la aparición de países emergentes (China, Brasil o India) con precios inferiores que obligan a países tradicionalmente comercializadores como España o Italia a competir en diferenciación, ya sea ofertando productos con valores añadidos como calidad y servicio, desarrollando nuevas líneas de productos y acabados o mediante la creación de una marca.

Tabla 3

Producción y exportación de granito en España (toneladas)

\begin{tabular}{cccccc}
\hline AÑOS & PRODUCCIÓN & EXPORTACIÓN & AÑOS & PRODUCCIÓN & EXPORTACIÓN \\
\hline 1993 & 1.170 .000 & 493.000 & 2001 & 1.535 .000 & 610.000 \\
\hline 1994 & 1.250 .000 & 581.000 & 2002 & 1.412 .000 & 585.000 \\
\hline 1995 & 1.375 .000 & 652.000 & 2003 & 1.385 .000 & 540.000 \\
\hline 1996 & 1.295 .000 & 603.000 & 2004 & 1.400 .000 & 535.000 \\
\hline 1997 & 1.174 .000 & 580.000 & 2005 & 1.519 .000 & 517.000 \\
\hline 1998 & 1.256 .000 & 548.000 & 2006 & 1.575 .000 & 567.000 \\
\hline 1999 & 1.555 .000 & 591.000 & 2007 & 1.559 .000 & 571.000 \\
\hline 2000 & 1.976 .000 & 638.000 & & & \\
\hline
\end{tabular}

Fuente: Roc Natural Stone in the world 2007

Dada la pequeña dimensión y el reducido número de empresas en el sector del granito puede que éstas no dispongan de suficientes recursos financieros como para crear una marca propia o que exista un gran número de oferentes con una escasa notoriedad y sin una posición dominante (Kapferer, 1992); en estos casos una marca paraguas que los reúna a todos puede ser una solución ${ }^{8}$ (Baker y Ballington, 2002; Lin y Chen, 2006; Valls, 1993). Esta marca respaldaría todos los productos y empresas, sin condicionar su desarrollo individual (Pappu, Quester y Cooksey, 2006) ${ }^{9}$.

\footnotetext{
${ }^{7}$ Turquía es un productor especializado en mármoles, con escasa presencia en granitos.

${ }^{8}$ La Federación Española de la Piedra Natural creó la imagen «Stone from Spain», como instrumento de identificación de los pabellones que organiza en las ferias internacionales con la participación de las empresas del sector.

${ }^{9}$ Algunos autores establecen diferencias entre la imagen de un país y la que pueda tener sobre el capital reputacional en los distintos sectores económicos e industriales. Así, cuando exista este último, tendrá una mayor influencia en las decisiones de compra que otras posibles creencias e imágenes del país (Cerviño, 2002). Por ello, el sector del granito puede buscar su propio posicionamiento.
} 
La marca paraguas serviría para desempeñar un papel diferenciador, donde el made in actuaría como un signo distintivo de un grupo de empresas cuyos productos presentan una serie de características distintas y específicas como: suelo, clima, saber-hacer, historia, etc. Se utilizaría como un símbolo de reconocimiento y pertenencia que aunaría las acciones realizadas para dar a conocer la calidad y la identidad de un producto y a su vez, obtener notoriedad y diferenciación ${ }^{10}$.

\section{ANÁLISIS EMPÍRICO DEL SECTOR DEL GRANITO}

Con el objeto de analizar la situación en el sector del granito realizamos un estudio exploratorio en el ámbito gallego que constituye el núcleo fundamental en España ${ }^{11}$. Para ello realizamos un censo de empresas recurriendo a diversas fuentes que, una vez cruzadas y depuradas, incluía un total de $48^{12}$. Entre ellas había empresas de muy diversa condición, desde explotaciones semiartesanales de marcado carácter local hasta empresas mucho más grandes, tecnificadas y con gran vocación exportadora. Como nuestro interés se orientaba hacia la construcción de una marca genérica optamos por centrarnos en las de una mayor proyección en su actuación; más representativas a los efectos del trabajo y con mayor sensibilidad hacia tales cuestiones.

El listado inicial se segmentó en base a las variables: volumen de facturación, número de trabajadores y pertenencia a alguna asociación del sector, descartándose 23 firmas de pequeña dimensión, con lo que se redujo el listado inicial a 25 empresas consideradas significativas para el trabajo. Posteriormente se concertaron entrevistas con los gerentes o directores comerciales, obteniéndose un índice de respuestas cercano al 50\%. La necesidad de alcanzar cierta representatividad y el reducido tamaño de la población objeto de estudio obligó a realizar sucesivas rondas de consulta, consiguiéndose finalmente 20 respuestas.

En una primera instancia, fruto del trabajo de campo, constatamos dos aspectos relevantes en el sector: 1) El predominio de la actividad de elaboración frente a la extracción. Un 70\% de las empresas se declaran transformadoras y, del 30\% restante, el 25\% combina extracción y elaboración; 2) El alto grado de internacionalización. Sólo el 25\% afirman que su principal mercado objetivo es el nacional, manifestando el resto una marcada propensión exportadora.

Se confirma así un aumento en la exportación de granito elaborado, línea maestra de los planes de dinamización del sector en los últimos años, que indica un creciente interés de las empresas por añadir valor a la producción y penetrar en mercados sofisticados.

Al preguntar por la utilidad de crear una marca (gráfico 1), solamente un $15 \%$ de las empresas encuestadas lo consideran poco interesante. Es más, algunas perciben un interés parcial, ligado exclusivamente a la función comercial, o entienden que la diferenciación va asociada a la variedad de la piedra que se comercializa. Sólo una sostiene que la competencia en el merca-

\footnotetext{
${ }^{10}$ El mármol de Carrara, el café de Colombia o las bananas Chiquita nos muestran ejemplos de commodities que han sabido aprovechar los beneficios de la marca y el made in.

${ }^{11}$ En España, Galicia es la región española con mayores recursos en cuanto a materias primas, variedad de productos y tradición en la explotación y tratamiento del granito. Más concretamente, la villa de Porriño es el centro puntero de extracción y elaboración de granito.

${ }^{12}$ Las principales fueron: la Asociación de Graniteros de Galicia y ARDÁN.
} 
do es vía precios, contexto en el que la marca no serviría para mejorar la posición competitiva del producto. Por el contrario, el $85 \%$ restante defiende el interés de una marca señalando motivos diversos, entre los que destaca el interés para la diferenciación del producto.

\section{Gráfico 1}

\section{Razones que justificarían la creación de una marca genérica (\%)}

$\square$ Control actividad sumergida

$\square$ Revalorizar producto

Cohesionar el sector

$\square$ Fomentar calidad

Reforzar competitividad Internacional

$\square$ Diferenciación producto

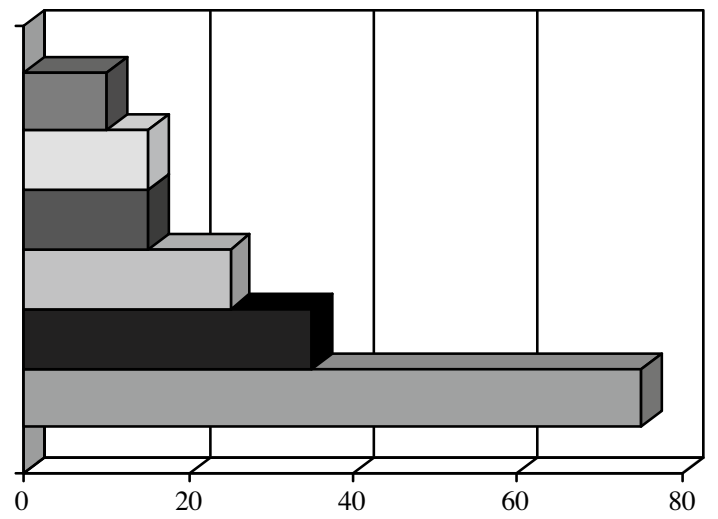

Ahora bien, para crear una marca para el granito es necesario buscar unas características que le aporten una personalidad propia y lo distingan de sus competidores. Con ese fin, se les pidió a las empresas que valorasen de 1 (nada importante) a 7 (muy importante) los factores sobre los que, según su opinión, se debería cimentar la competitividad del granito gallego en los mercados exteriores (gráfico 2).

Gráfico 2

Factores de competitividad del granito gallego en los mercados exteriores

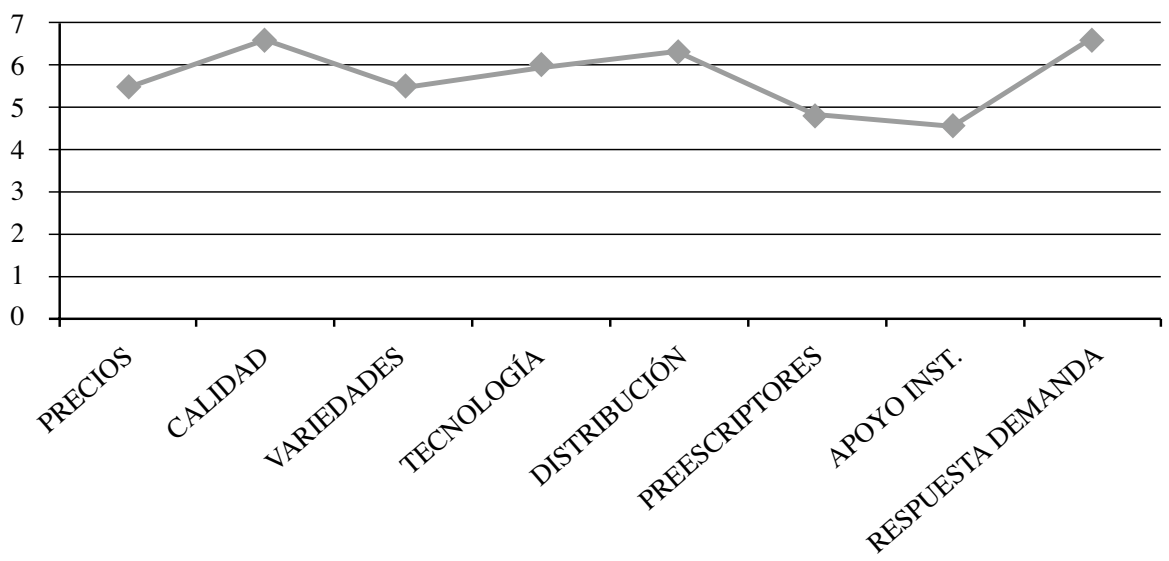


Los dos factores más valorados son la capacidad de respuesta a la demanda y la ca$\operatorname{lidad}^{13}$, seguidos de cerca por el dominio de los canales de distribución, la tecnología y las variedades apreciadas o exclusivas. El acceso a prescriptores y el apoyo brindado por organismos e instituciones públicas resultan relativamente poco valorados, a pesar de que son aspectos sobre los que suele incidirse en los estudios realizados sobre el sector. Los dos atributos más valorados se deberían utilizar para construir la imagen de marca, siempre y cuando los diferencien de los competidores internacionales.

Por ello, una vez identificados los factores que se consideran importantes a escala internacional, hemos tratado de determinar en cuáles piensan las empresas que destacan sus competidores. Con ese fin les preguntamos por la importancia de los factores de competitividad en los países emergentes (Brasil, China, India y Sudáfrica), y en Italia (país de referencia en el sector) (gráfico 3).

\section{Gráfico 3}

\section{Perfiles percibidos de los competidores internacionales}

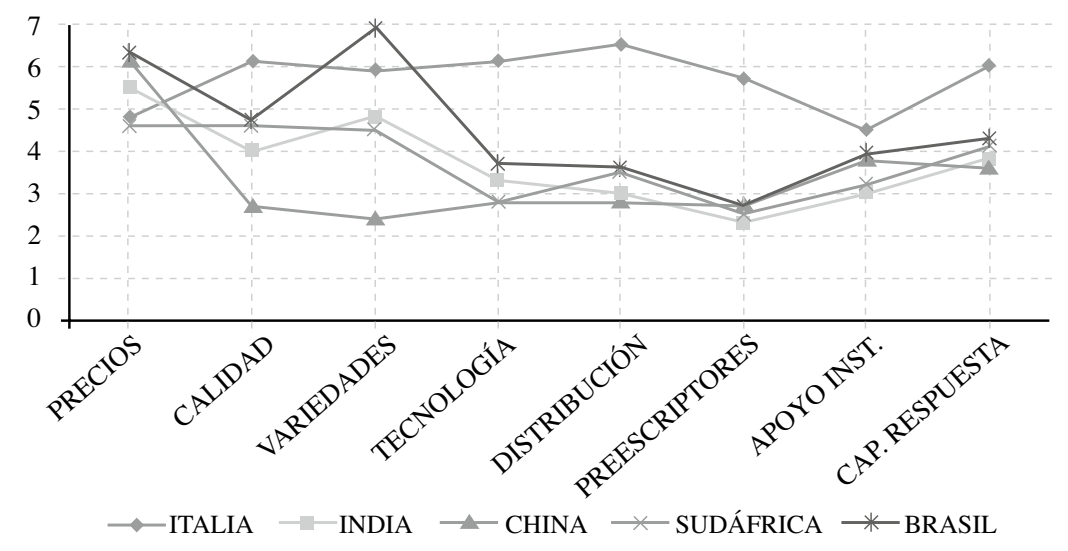

Podemos observar como Italia se erige como el líder del sector, con puntuaciones muy altas en todos los factores, excepto en precios y variedad. En relación al primero, Brasil, China e India son más competitivos. No obstante, llama la atención la poca diferencia que existe al respecto entre los países. En cuanto a la variedad del producto, Brasil es el país mejor valorado. Sus granitos, de tonalidades azules, verdes y amarillas, no existen en otros países, donde la piedra presenta coloraciones grises y rosas. India también tiene variedades muy distintas, con granitos de color rojo, negro o verde, entre otros.

India y Sudáfrica presentan perfiles similares, aunque el primero ofrece una imagen de precios más bajos pero con inferiores niveles de calidad en el producto. Ambos países

\footnotetext{
${ }^{13}$ La calidad de la piedra está condicionada por las actividades de extracción, elaboración y colocación final. Aunque es en esta última etapa donde se aprecian realmente las cualidades y prestaciones del granito como roca ornamental, la calidad está en relación directa con las características de la piedra en el yacimiento, como el color, la textura o la fracturación, singularidades que condicionarán la respuesta del material una vez colocado.
} 
presentan limitaciones en su capacidad de acceso al mercado y en el potencial para dar servicio a demandas complejas.

Por último y a pesar de que China es el país que ha experimentado un mayor crecimiento en la exportación y producción de piedra a nivel mundial, su perfil se sitúa por debajo del resto de países en la mayoría de los factores, fundamentalmente en aspectos como los niveles de calidad y el grado de apreciación de las variedades comercializadas. También se refleja la visión de una relativa agresividad en precios, así como una capacidad de respuesta a los requerimientos de los clientes más limitada.

En resumen, comparando las gráficos 2 y 3 , podemos comprobar que el granito gallego destaca frente a sus competidores en calidad y capacidad de respuesta a la demanda, por lo que estos atributos, como se ha comentado anteriormente, se pueden utilizar para crear una marca paraguas del sector.

\section{CONCLUSIONES}

España ha perdido peso en el mercado mundial del granito debido fundamentalmente a la competencia de los países emergentes que ofrecen unos precios inferiores, como consecuencia, deberá potenciar sus fortalezas posicionando correctamente el producto y para ello la marca constituye una potente herramienta de identificación y diferenciación.

Para la creación de una marca genérica resulta necesario crear un clima de confianza entre las empresas, intentando conseguir que todas sean conscientes de que saldrán beneficiadas con una acción colectiva que permita crear una buena imagen del granito. Además, este esfuerzo debe realizarse colaborando con las instituciones puesto que la actuación de empresas y administración ha de reforzarse mutuamente. Teniendo en cuenta que el reconocimiento del granito en los mercados internacionales se articula en función del país de procedencia y no en base a las empresas que lo transforman y comercializan, consideramos que este tipo de actuación conjunta resulta no sólo más adecuada, sino imprescindible.

Por otra parte, de cara a la venta de los productos en el mercado exterior, el made in es un valioso elemento de referencia para el consumidor, siempre y cuando la imagen del país sea positiva, por lo que puede ser utilizado como un componente de la imagen de esa marca. En el caso del granito, el país de origen constituye un punto de referencia básico para conocer las características del mismo, ya que se trata de un producto genérico y de un sector globalizado, tal y como ponen de manifiesto las cifras relativas a su comercio exterior, la presencia de países procedentes de los cinco continentes en las ferias internacionales o la difusión acelerada de las tecnologías de extracción, corte y transformación.

Las empresas del sector muestran una actitud positiva hacia la creación de una marca paraguas y aportan como principales atributos comunes a resaltar en su producto: la calidad y la capacidad de respuesta a la demanda, aspectos en los que destacan sobre sus principales competidores internacionales. En una comparación con estos países se comprueba que salvo Italia, país altamente valorado en todos los factores de competitividad, el resto de contendientes centran sus fortalezas en factores distintos, como los precios y las variedades de la piedra. 


\section{REFERENCIAS BIBLIOGRÁFICAS}

AAKER, D.A. (1994): Gestión del valor de la marca. Capitalizar el valor de la marca, Madrid, Díaz de Santos S.A.

AAKER, D.A. (1996): Construir marcas poderosas, Barcelona, Ediciones gestión 2000.

AGRAWAL, J. Y KAMAKURA, W.A. (1999): «Country of origin: a competitive advantage», International Journal of Research in Marketing, Vol. 16, pp. 255-267.

ÁLVAREZ DEL BLANCO, R.M. (2000): «País de origen como estereotipo: ¿ventaja competitiva para la marca?», Harvard Deusto Business Review, Nº8, sep-oct, p. 38-54.

ÁLVAREZ DEL BLANCO, R.M. (2002): «Imagen país y marca. La marca España», en Las marcas renombradas españolas, Mc Graw Hill, Madrid.

ARGAWAL, S. Y SIKRI, S. (1996): «Country image: Consumer evaluation of product category extensions», International Marketing Review, Vol. 13, № 4, pp. 23-39.

AXELROD, J.N. Y WYBENGA, H. (1985): «Perceptions that motivate purchase», Journal of Advertising Research, Junio-Julio, pp. 19-22.

BAKER, M.J. Y BALLINGTON, L. (2002): «Country of origin as a source of competitive advantage», Journal of Strategic Management, Vol. 10, pp. 157-168.

BIEL, A.L. (1992): «How brand image drives brand equity», Journal of Advertising Research, Vol. 32, $\mathrm{N}^{\circ} 6$, pp. RC6-RC12.

BIGNÉ, E.; PERIS, S.M. Y NEWMAN, K. (1993): «La imagen de los productos fabricados en España», Información Comercial Española, $\mathrm{N}^{\circ}$ 722, pp. 49-60.

BILKEY, W.J. Y NES, E. (1982): «Country of origin effects on product evaluations», Journal of International Business Studies, Vol. 13, № 1, pp. 89-99.

CERVIÑO FERNÁNDEZ, J. (2002): Marcas internacionales. Cómo crearlas y gestionarlas, Madrid, Pirámide.

CERVIÑO, J. Y RIVERA, J. (2007): «La globalización de las marcas españolas: liderazgo y notoriedad internacional», ICE, $\mathrm{N}^{\circ} 839$, pp.121-139.

CORDELL, V. (1992): «Effects on consumer preferences for foreign sourced products», Journal of International Business Studies, Vol. 23, N ${ }^{\circ} 2$, pp. 251-269.

DEHESA, G. (2005): «Marcas e imagen país cuestión de sinergias», en Marca y cultura. De Altamira a las marcas renombradas, Asociación de marcas renombradas españolas.

ERICKSON, G.M.; JOHANSSON, J.K. Y CHAO, P. (1984): «Image variables in multiattribute product evaluations: Country of origin effects», Journal of Consumer Research, Vol. 11, septiembre, pp. 649-699.

FERNÁNDEZ MADRID, J. (1996): Manual del granito para arquitectos, Santiago de Compostela, Asociación Gallega de Graniteros D.L.

GONZÁLEZ HERNÁNDEZ, M.J. (1997): «Del made in al etnocentrismo del consumidor: Un estudio exploratorio de los factores influyentes en la imagen de los productos», Actas del IX encuentro de profesores universitarios de marketing, Editorial ESIC, Murcia.

HAN, C. (1989): «Country image: halo o summary construct?», Journal of Marketing Research, Vol. 26, No 2 (mayo), pp. 222-229

HAN, C. (1990): «Testing de role of country image in consumer choice behavior», European Journal of Marketing, Vol. 24, $\mathrm{N}^{\circ}$ 6, pp. 24-40.

HAN, C. Y TERPSTRA, V. (1988): «Country of origin effects for uni-national and bi-national products», Journal of International Business Studies, Vol.19, ํ2, pp. 235-255.

HEIMBACH, A.E.; JOHANSSON, J. K. Y MACLACHLAN, D.L. (1989): «Product familiarity, information processing and country of origin cues», Advances in Consumer Research, Vol. 16, $\mathrm{N}^{\circ} 1$, pp. 460-467.

HONG, S. Y WYER, R. (1989): «Effects of country of origin and product attribute information on 
product evaluation: an information processing perspective», Journal of Consumer Research, Vol. 16, No 2, septiembre, pp. 175-187.

HOOLEY, G.J.; SHIPLEY, D. Y KRIEGER, N. (1988): «A method for modelling consumer perceptions of country of origin», International Marketing Review, Vol. 5, otoño, pp. 67-76.

IBAÑEZ ZAPATA, J.A. Y MONTORO RÍOS, F.J. (1996): « Análisis de la importancia del atributo país de origen en la valoración de las entidades financieras: una propuesta metodológica», Libro de ponencias del X Congreso Nacional y VI Congreso Hispano Francés de AEDEM, Granada, Vol. IB, pp. 605-625.

JOHANSSON, J.K.; DOUGLAS, S.P. Y NONAKA, I. (1985): «Assessing the impact of country of origin on product evaluations: a new methodological perspective», Journal of Marketing Research, Vol. 22, No 4, pp.388-396.

KAYNAK, E. Y CAVUSGIL, S.T. (1983): «Consumer attitudes towards products of foreign origin: do they vary across product class?», International Journal of Advertising, Vol. 2, $\mathrm{N}^{\circ} 2$, pp. $147-$ 157.

KAPFERER, J-N.(1992): La marca, capital de la empresa. Principios y control de su gestión, Bilbao, Deusto S. A.

KIM, CH. Y CHUNG, J. (1997): «Brand popularity, country image and market share: an empirical study», Journal of International Business Studies, Vol.28, №2, pp. 361-386.

KNIGHT, G, (1999): «Consumer preferences for foreign and domestic products», Journal of Consumer Marketing, Vol. 16, № 2, pp. 151-162.

LAMPERT, S.I. Y JAFFE, E.D. (1998): «A dynamic approach to country of origin effect», European Journal of Marketing, Vol. 32, $\mathrm{N}^{\mathrm{o}} 1 / 2$, pp. 61-78.

LAROCHE, M.; PAPADOPOULOS, N.; HESLOP, L.A. Y MOURALI, M. (2005): »The influence of country image structure on consumer evaluations of foreign products», International Marketing Review, Vol. 22, № 1, pp. 96-115.

LIN, L.Y. Y CHEN, C.S. (2006): «The influence of the country of origin image, product knowledge and product involvement on consumer purchase decisions: an empirical study of insurance and catering services», Journal of Consumer Marketing, Vol.23, №5, pp.248-265.

LÓPEZ JIMENO, C. (ED.) (1995): Manual de rocas ornamentales: prospección, explotación, elaboración y colocación, Entorno Gráfico, Madrid.

MACKENZIE, S.B.; LUTZ, R.J Y BELCH, G.E. (1986):»The role of attitude toward de AD as a mediator of advertising effectiveness: A test of competing explanations», Journal of Marketing Research, Vol. XXIII, Mayo, pp. 130-143.

MARTÍNEZ SENRA, A.I. Y GARCÍA RODRÍGUEZ, M.J. (2001): «Las ventajas del efecto made in: una aplicación al granito gallego», Revista Galega de Economía, Vol. 10, № 1, pp.279-294.

MELLA MÁRQUEZ, X.M. (COORD.) (1994): Plan estratégico de Vigo y su área de influencia, Consorcio de la Zona Franca de Vigo.

MIQUEL PERIS, S.; BIGNÉ ALCAÑIZ, J.E.; FRASQUET DEL TORO, M.; KÜSTER BOLUDA, I. Y VILA LÓPEZ, N. (1996): «El efecto del país de origen en la formación de actitudes», en T. Luque Martínez (ed.), La empresa en una economía globalizada: Retos y cambios, Vol. 1A, Málaga, pp. 31-44.

MONTAÑA. J. (1993): «La imagen exterior del diseño español y su influencia sobre las marcas», Información Comercial Española, $\mathrm{N}^{\circ} 722$, octubre, pp. 89-95.

NISS, H. (1996): «Country of origin marketing over the product life cycle. A Danish case study», European Journal of Marketing, Vol. 30, No 3, pp. 6-22.

OPORTO DEL OLMO, A. (1993): «La imagen exterior de España y el marketing de país», Información Comercial Española, $\mathrm{N}^{\mathrm{o}}$ 722, octubre, pp. 27-33.

PAPADOPOULOS, N.G. Y HESLOP, L.A. (2002): «Country equity and country branding: Problems and prospects», The Journal of Brand Management, Vol. 9, No 4-5, pp. 294-314.

PAPPU, R.; QUESTER, P.G. Y COOKSEY, R.W. (2006): «Consumer-based brand equity and coun- 
try of origin relationships», European Journal of Marketing, Vol. 40, № 5/6, pp. 696-717.

PARAMESWARAN, R. Y PISHARODI, R.M. (1994): «Facets of country of origin image: an empirical assessment», Journal of Advertising, Vol.13, Nº 1 , marzo, pp.43-56.

RICE, C. (1993): Consumer behaviour. Behavioural aspects of marketing, Butterworth-Heinemann, Oxford.

ROC MÁQUINA : ANUARIOS 1993-2007.

RODRÍGUEZ GONZÁLEZ, X. A. Y GONZÁLEZ MURIAS, P. (2001): La industria del granito en el siglo XXI: realidad actual y estrategias económicas de futuro, Santiago de Compostela, Asociación Hispalink-Galicia.

SÁNCHEZ FRANCO, M.J. Y VILLAREJO RAMOS, A.F. (1996): «La imagen de marca: una aproximación a las estrategias de internacionalización», en T. Luque Martínez (ed.), La empresa en una economía globalizada: Retos y cambios, Vol. 1B, Málaga, pp. 731-745.

VALLS, J-F. (1992): La imagen de marca de los países, Madrid, McGraw-Hill.

VALLS, J-F. (1993): «La imagen de marca de España», Información Comercial Española, $\mathrm{N}^{\circ} 722$, pp.61-70. 


\section{Gipuzkoaren kanpo harremanak, esku onetan.}

Las relaciones externas de Gipuzkoa, en buenas manos.

Les relations externes de Gipuzkoa, en bonnes mains.

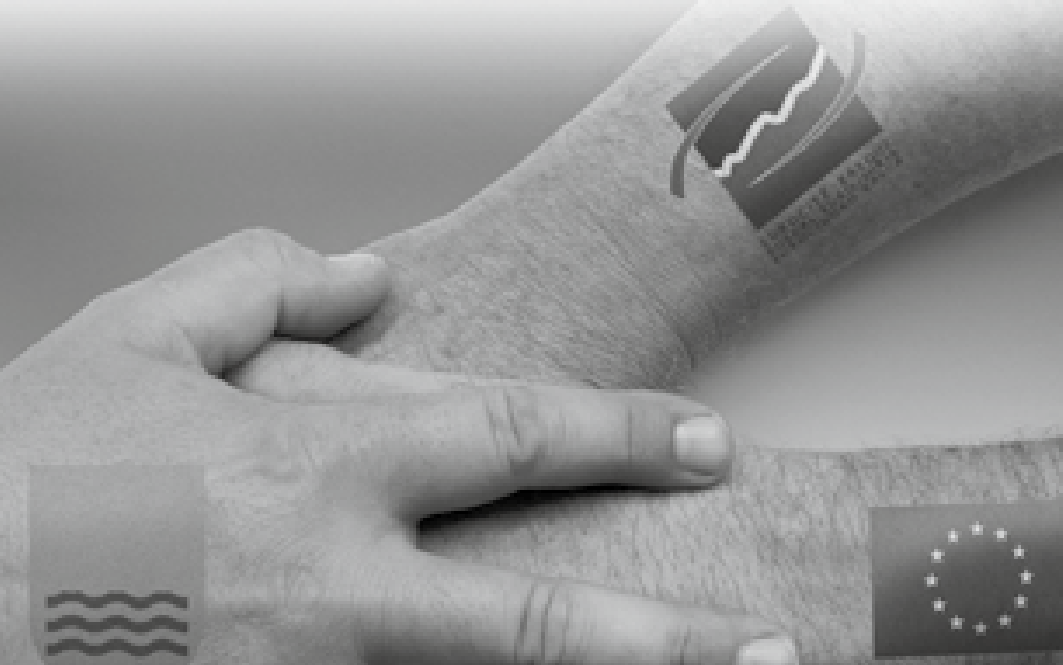

